\title{
SHORT REPORT \\ Use of sentinel chickens to study the transmission dynamics of West Nile virus in a sahelian ecosystem
}

\author{
V. CHEVALIER ${ }^{1 *}$, R. LANCELOT ${ }^{2}$, A. DIAÏTE ${ }^{3}$, B. MONDET ${ }^{4}$ \\ AND X. DE LAMBALLERIE \\ ${ }^{1}$ CIRAD, UPR 'Epidemiology and ecology, Campus International de Baillarguet, Montpellier, France \\ ${ }^{2}$ CIRAD, Campus International de Baillarguet, Montpellier, France \\ ${ }^{3}$ ISRA, Laboratoire National d'Elevage et de Recherche Vétérinaire, Dakar-Hann, Sénégal \\ ${ }^{4}$ IRD, UR 178, Dakar-Hann, Sénégal \\ ${ }^{5}$ Unité des Virus Émergents, Faculté de Médecine, Université de la Méditerranée, Marseille, France
}

(Accepted 25 April 2007)

\section{SUMMARY}

During the 2003 rainy season, a follow-up survey in sentinel chickens was undertaken to assess the seasonal transmission of West Nile virus (WNV) in a sahelian ecosystem: the Ferlo (Senegal). The estimated incidence rate in chickens was $14 \%$ (95\% CI 7-29), with a very low level of transmission between July and September, and a transmission peak occurring between September and October. Comparing these results with the estimate obtained from a previous transversal serological study performed on horses the same year in the same area, the relevance of sentinel chickens in estimating the WNV transmission rate is highlighted. Conventionally adult mosquito populations disappear during the dry season but WN disease was shown to be endemic in the study area. The mechanisms of virus maintenance are discussed.

West Nile fever (WNF) is a mosquito-borne disease caused by a flavivirus (genus Flavivirus, family Flaviviridae). The transmission cycle involves birds (amplifying hosts) and mosquitoes which may also transmit West Nile virus (WNV) to other species, including humans and horses. Most infections in humans are asymptomatic or lead to a non-specific mild febrile syndrome. Rare, albeit severe, neurological forms have been reported in humans during the last two decades [1] and heavy losses were observed in naive horse populations in different parts of the world.

WNF has often been associated with wetlands. However, the virus is also observed in drier areas. For example, it was isolated from Aedes and Culex

\footnotetext{
* Author for correspondence: Dr V. Chevalier, CIRAD, UPR 'Epidemiology and ecology', Campus International de Baillarguet TA 30/G, 34398 Montpellier, France. (Email: chevalier@cirad.fr)
}

mosquitoes in the Ferlo (Senegal) [1] between 1988 and 1995. A serological survey in horses showed that the infection was endemic in this sahelian ecosystem [2]. We report here the results of a follow-up survey in sentinel chicken flocks implemented in the Ferlo region to assess the seasonal transmission dynamics of WNV.

The study was undertaken in Barkedji $\left(14^{\circ} 52^{\prime} \mathrm{W}\right.$, $\left.15^{\circ} 16^{\prime} \mathrm{N}\right)$, a village located in the Ferlo. The climate is hot and dry with an annual rainfall ranging from 300 to $500 \mathrm{~mm}$, occurring between July and September. The Ferlo was an effluent of the Senegal River which dried up in 2000 B.C. During the rainy season, temporary ponds appear in the Ferlo bed: they start to fill up in July and remain flooded until October-December.

These ponds and their environment constitute a favourable ecosystem for WNV. Culex and Aedes are the dominant mosquito genera and are considered the 
most likely vectors of WNV [1]. Many birds are attracted by the water and thus contribute to the maintenance of the epidemiological cycle. Nomadic ruminant farmers (cattle, sheep and goats) frequently come into close association with ponds. They rear horses for transportation and agricultural work. Humans and horses live in close proximity, thus sharing common exposure conditions to WNV.

For the purpose of the study, daily rainfall was recorded with a rain gauge located in Barkedji.

Six cohorts of 1-day-old chicks were exposed to WNV from July to December 2003. They were purchased in a commercial hatchery in Dakar and settled in hen houses built for the purpose of the survey. Chickens were wing-tagged and received preventive treatment against common diseases (Gumboro, Newcastle, coccidiosis). Three cohorts were established, two on 15 July and one on 15 August. Chickens were sampled on 1 October and 1 November. Three more cohorts were settled on 1 October. Chickens from the first cohort were sampled on 1 November and 1 December. Chickens from the second cohort were sampled on 1 November. Chickens from the third cohort were only sampled on 1 December (Fig.). Chickens that tested positive on the first sampling occasion were kept and sampled later to rule out the possibility of maternal antibodies.

Chicken sera were tested for immunoglobulins $M$ (IgM) using an immunocapture enzyme-linked immunosorbent assay (ELISA). The test was adapted from a previously described method [3] by using (i) microplates coated with anti-horse IgM or antichicken IgM antibodies, and (ii) WNV antigen and anti-WNV mouse polyclonal, hyperimmune ascites fluid prepared from the prototype strain Eg-101. Threshold value was defined as the mean optical density of negatives plus three standard deviations. Positive results were confirmed using the plaque reduction neutralization test (PRNT) following a modified procedure [4]. Sera were inactivated at $56^{\circ} \mathrm{C}$ for $30 \mathrm{~min}$. Two-fold dilutions were made from $1 / 10$ to $1 / 320$. Serum dilutions and virus [containing 50 plaque-forming units of the equine New York 1999 WNV strain (NY99-eqhs) for the latter] were mixed and incubated at $37^{\circ} \mathrm{C}$ for $1 \mathrm{~h}$. The mixture was added to human SW13 cell monolayers and incubated at $37^{\circ} \mathrm{C}$ for $1 \mathrm{~h}$. Agarose was added and plates were incubated for 5 days at $37^{\circ} \mathrm{C}$. Cells were fixed with formaldehyde and stained with Naphthalene Black. Plaques were then counted. A serum was considered positive whenever both (i) a $90 \%$ reduction of the

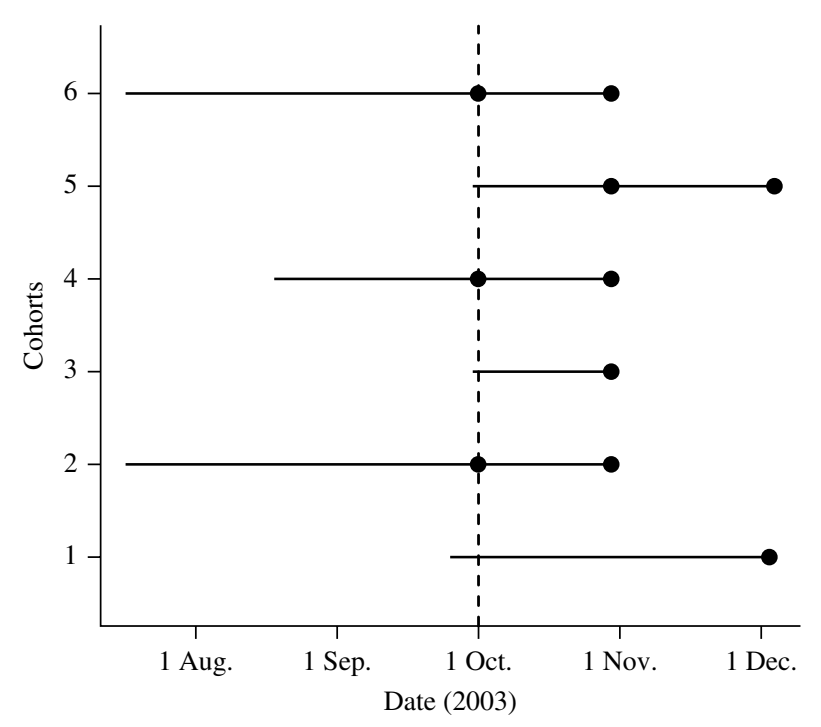

Fig. Survey design to study the serological incidence in six cohorts of chicks exposed to the West Nile fever virus in Barkedji (Senegal) during the rainy season, 2003. Sampling occasion.

number of plaques at the $1 / 10$ dilution and (ii) a $50 \%$ reduction of the number of plaques at the $1 / 40$ dilution were observed. This constitutes stringent interpretation criteria.

The survey period was divided into two intervals: July-September and October-November. These intervals were chosen according to the predominance of the two main genera of mosquitoes during the rainy season in Barkedji: Aedes vexans at the beginning and Culex poicilipes at the end of the rainy season. In fact, Culex eggs and larvae cannot survive in draining ponds. Usually, Culex populations peak several weeks after ponds are watered, and when rainfalls are heavy and regular. The eggs of $A$. vexans are laid on the wet soil of the pond banks, and their desiccation is needed before they hatch when they are watered again. Aedes populations peak at the beginning of the rainy season, when rainfalls are irregular and allow ponds to dry [5].

The annual cumulative rainfall in 2003 was $379 \mathrm{~mm}$, showing a $5 \%$ deficit compared to the 1960-1990 annual average.

Mortality causes were not investigated in chickens. Previous studies showed that chickens are not clinically affected by WNV [6]. We therefore assumed that mortality was not related to WNV infection. The observed incidence rate $I_{i, t}$ in cohort $i(i=\{1,6\})$ during interval $t(t=\{1,2\})$ was computed as $I_{i, t}=c_{i, t} /$ $\left(n_{i, t}-0 \cdot 5 * m_{i, t}\right)$, where $c_{i, t}$ was the count of new serological cases in cohort $i$ during interval $t ; n_{i, t}$ was the 
Table. Serological incidence of West Nile virus antibodies in six cohorts of sentinel chickens in Barkedji (Senegal) during the rainy season 2003

\begin{tabular}{llccc}
\hline \hline Cohort & Period & Initial size & Deaths & Incidence \\
\hline 1 & Oct.-Nov. & 50 & 17 & 1 \\
2 & July-Sep. & 50 & 8 & 0 \\
2 & Oct.-Nov. & 42 & 1 & 11 \\
3 & Oct.-Nov. & 50 & 44 & 0 \\
4 & July-Sep. & 100 & 15 & 0 \\
4 & Oct.-Nov. & 85 & 2 & 2 \\
5 & Oct.-Nov. & 50 & 20 & 3 \\
5 & Oct.-Nov. & 50 & 20 & 6 \\
6 & July-Sep. & 50 & 16 & 1 \\
6 & Oct.-Nov. & 34 & 3 & 14 \\
\hline \hline
\end{tabular}

number of chickens in cohort $i$ at the beginning of interval $t$, and $m_{i, t}$ was the number of deaths in cohort $i$ during interval $t$.

Because different cohorts were used and repeat observations were made on chickens in the cohorts, overdispersion was likely to occur (variance greater than the expected binomial variance). A beta-binomial logistic regression model was used to overcome this problem and estimate interval-specific incidence rates $I_{1}$ and $I_{2}$. The overall incidence from July to November was computed as $I=1-\left(1-I_{1}\right) *\left(1-I_{2}\right)$.

From July to September, 200 chickens were involved in the follow-up survey (Table). The observed mortality rate was $20 \%$. Thus, 161 chickens were sampled and their sera were tested. A single serum tested positive: the observed incidence rate was $1 \%$. From October to November, 361 chickens (including those surviving and still seronegative for WNV after the first interval) were exposed to transmission risk (Table). The observed mortality rate was $30 \%$, therefore 254 sera were collected and analysed. The serological incidence was 38 during this second interval, i.e. an observed incidence rate of $15 \%$. Interval-specific incidence rates estimated with the beta-binomial logistic regression model were $1 \%$ (95\% CI 0-4) and 14\% (95\% CI 6-28), respectively. The overall estimated serological incidence from July to November was $14 \%$ (95\% CI 7-29).

Thus, WNV circulated in Barkedji during the 2003 rainy season. The annual cumulative rainfall in 2003 showed a deficit of 5\% compared to the 1960-1990 annual average [5], suggesting that WNV may circulate even under very dry climatic conditions.

The observed incidence rate in chickens was lower than that estimated in horses in the same area in 2003
(14\% vs. $22 \%$ ) [2]. Moreover, the incidence rate was lower than the rates reported in 1997 in 160 sentinel chickens scattered throughout the city of Bucharest (Romania), after the 1996 outbreak: 16-40\% [7]. Previous studies suggested that sentinel chickens should be sampled weekly for optimal detection of antibodies, as some animals exhibited a low IgM response [6]. With a 1-month interval between successive samples and a high stringency threshold for antibody detection, WNV serological incidence might have been underestimated in our study. Nevertheless, WNV undoubtedly circulated in the Ferlo region. High chicken mortality rates were probably related to the climatic conditions in Barkedji: hot and dry at the beginning of the rainy season, hot and wet with storms in the middle and at the end of the rainy season.

Chicken and horse incidence rates were difficult to compare. The serological incidence rate in chickens was probably more reliable than the serological incidence rate in horses, because the former was directly observed while the latter was computed and relied on several assumptions. In addition, C. poicilipes is mainly ornithophilic, and thus more attracted to chickens than horses. A. vexans, which is mammophilic, is probably involved in the transmission to horses. The impact of WN diseases on human and horse populations should be evaluated.

According to chicken data, WNV transmission began as soon as the rainy season began, when the Aedes population was dominant and the Culex population at a very low level [5]. This result corroborated with that from the horse survey: WN-reactive IgM were detected in horses sampled in July [2]. WNV has often been isolated from $A$. vexans in the Ferlo area $[1,10]$. Even if it was shown that this species was not an efficient WN vector [8], A. vexans may compensate for its lack of effectiveness by its abundance, and act as an early initiator and a bridge vector of the $\mathrm{WN}$ cycle in this region. Then the transmission cycle is amplified to reach a maximum intensity at the end of the rainy season when the Culex population is dominant and the Aedes population at a low level [5]. Therefore, Culex mosquitoes were probably involved in the amplification of the epidemiological cycle at the end of the rainy season in 2003. The abundance of Culex mosquitoes is subject to large annual variations [9, 10]. In fact, eggs and larvae of this genus do not survive during the dry periods [11]. Several rainfall shortages were observed during the rainy season of 2002, resulting in successive cycles of flooding and 
draining of the ponds. Therefore, Culex abundance was low and $A$. vexans - whose biology and ecology are well-adapted to this rainfall pattern, remained the dominant species throughout the rainy season [12]. These variations may cause annual changes in the incidence of WNF.

The processes that allow the maintenance of the virus in arid areas remain unknown. In South Africa, where the annual rainfall level is below $500 \mathrm{~mm}$, the disease is endemic [13]. The virus could be maintained during the dry season via a low-level circulation between wild birds and Culex univittatus [14]. In the Ferlo area, whether the virus is maintained on ticks, batraciens or overwintering Culex, or is regularly introduced via migrating birds remains unknown.

Bird-borne dissemination appears to be the most likely hypothesis. However, this is difficult to demonstrate: thousands of bird captures, blood samples and laboratory analyses would be needed to assess the transmission risk of WNV between distant ecosystems. Further studies are needed to investigate the maintenance mechanisms of the virus in the Ferlo area during the dry season.

\section{ACKNOWLEDGEMENTS}

We thank M. Lesnoff, R. Pin, D. A. Sow, T. Manga, the horse owners and local authorities of Barkedji. The study was supported by grant 'ACI Écologie Quantitative' funded by the Ministry of Research, and grant 'CORUS' no. 02317057, funded by the Ministry of Foreign Affairs.

\section{DECLARATION OF INTEREST}

None.

\section{REFERENCES}

1. Murgue B, Zeller H, Deubel V. The ecology and epidemiology of West Nile Virus in Africa, Europe and Asia. Current Topics in Microbiology and Immunology 2002; 267: 195-221.
2. Chevalier V, et al. Serological assessment of West Nile fever virus activity in the pastoral system of Ferlo (Senegal). Annals of the New York Academy of Sciences 2006; 1081: 216-225.

3. Charrel R, et al. Prevalence of antibody against West Nile virus in volunteer blood donors living in southeastern France. Transfusion 2001; 41: 1320-1321.

4. Buckley A, et al. A. Serological evidence of West Nile virus, Usutu virus and Sindbis virus infection of birds in the UK. Journal of General Virology 2003; 84: 2807 2817.

5. Mondet B, et al. Rainfall patterns and population dynamics of Aedes (Aedimorphus) vexans arabiensis Patton, 1905 (Diptera, Culicidae), a potential vector of Rift Valley fever virus in Senegal. Journal of Vector Ecology 2005; 30: 102-110.

6. Johnson A, et al. Detection of anti-West Nile virus Immunoglobulin in chicken serum by an enzyme-linked immunosorbent assay. Journal of Clinical Microbiology 2003; 41: 2002-2007.

7. Cernescu C, et al. Continued transmission of West Nile virus to humans in southeastern Romania, 1997-1998. Journal of Infectious Diseases 2000; 181: $710-712$.

8. Turell MJ, Sardelis DJD, O'Guinn ML. Potential north american vectors of West Nile virus. Annals of the New York Academy of Sciences 2001; 951: 317-324.

9. Traore-Lamizana M, et al. Isolations of West Nile and Bagaza viruses from mosquitoes (Diptera: Culicidae) in central Senegal (Ferlo). Journal of Medical Entomology 1994; 31: 934-938.

10. Mondet B, et al. Relationship between rainfall pattern and the risk of virus transmission by mosquitoes: example of Rift Valley fever in the Ferlo area (Senegal) [in French]. Environment, Risk and Health 2005; 4: 125-129.

11. Beaty BJ, Marquardt WC. The Biology of Disease Vectors, 1996. Niwot: University Press of Colorado, $632 \mathrm{pp}$.

12. Chevalier V, et al. Exposure of sheep to mosquito bites: possible consequences for the transmission risk of Rift Valley fever in Senegal. Medical and Veterinary Entomology 2004; 18 : 247-255.

13. Jupp PG. The ecology of West Nile virus in South Africa and the occurence of outbreaks in humans. Annals of the New York Academy of Sciences 2001; 951: 143-152.

14. McIntosh B, Jupp PG, Dos Santos I. Infection by Sinbis and West Nile viruses in wild populations of Culex (Culex) univittatus Theobald (Diptera: Culicidae) in South Africa, Africa. Journal of the Entomological Society of Southern Africa 1978; 4: 57-61. 